\title{
Cifras de tensión arterial y factores asociados en niños de edad escolar de Mérida, Yucatán
} Blood pressure levels and associated factors in school
children from Merida, Yucatan

\author{
José J. Pérez-Miss ${ }^{1 *}$, FAnny C. VÁzquez-Encalada ${ }^{1}$, Lidia Moreno-Macías ${ }^{1}$, Rossana de F. Cuevas-Ferrera ${ }^{2}$, \\ Azalia G. KÚ-Doporto ${ }^{3}$, Delia J. SANSORes-España ${ }^{4}$ y Ligia del C. Vera-GamboA ${ }^{5}$
}

${ }^{1}$ Facultad de Medicina, Universidad Autónoma de Yucatán; ${ }^{2}$ Facultad de Psicología, Coordinación Laboratorio de Deporte, Universidad Autónoma de Yucatán; ${ }^{3}$ Subdirección de Salud, Ayuntamiento de Mérida; ${ }^{4}$ Escuela de la Salud, Coordinación de Posgrados en Nutrición, Universidad Modelo Mérida; ${ }^{5}$ Centro de Investigaciones Regionales "Dr. Hideyo Noguchi", Universidad Autónoma de Yucatán. Yucatán, México

\section{RESUMEN}

\begin{abstract}
Antecedentes: Diversos estudios han demostrado el impacto negativo del sobrepeso y la obesidad en la salud, específicamente la hipertensión, sin embargo, poco se ha estudiado en la edad pediátrica. Objetivo: Conocer la asociación del estado nutricional, dieta y actividad física con las cifras de presión arterial en niños escolares de Mérida, Yucatán. Material y métodos: Estudio transversal, observacional, analítico y prospectivo en 297 escolares, previo consentimiento informado de los padres; se valoró: estado nutricional, presión arterial, dieta consumida y actividad física realizada. Pruebas de chi cuadrada y razón de momios (RM) se utilizaron para búsqueda de asociación y riesgo. Resultados: La prevalencia de sobrepeso y obesidad fue del $47.2 \%$, el $15.5 \%$ presentó presión arterial elevada, el 52.5\% no realizaban actividad física según las recomendaciones de la Organización Mundial de la Salud y la dieta no se apegó a las recomendaciones para población mexicana. El sexo y el estado nutricional mostraron asociación significativa ( $p<0.005)$, así como la presión arterial y el estado nutricional $(p<0.05)$ y una RM de 6.9 (intervalo de confianza: 3.1-15.4). Conclusiones: Los niños con sobrepeso/obesidad presentaron asociación significativa y un riesgo mayor de presentar hipertensión arterial que los niños eutróficos, por lo que la prevención primaria debe iniciar desde la infancia.
\end{abstract}

Palabras clave: Estado nutricional. Tensión arterial. Dieta. Actividad física. Niños.

\section{ABSTRACT}

\begin{abstract}
Background: Diverse studies have shown the negative impact of overweight and obesity on people's health, specifically the hypertension. However, this problematic has not been deeply studied in the pediatric age. Objective: To know the association between the nutritional status, diet and physical activity with the blood pressure levels in school children from Mérida, Yucatán. Methods: Cross-sectional, observational, analytical, and prospective study including 297 schoolchildren, previous parental informed consent, nutritional status, blood pressure, diet consumed, and physical activity performed were assesed. Chi-square test and odds ratio were used to search for association and risk. Results: A prevalence of overweight and obesity of $47.2 \%$ was found, $15.5 \%$ had some degree of high blood pressure, $52.5 \%$ do not perform physical activity according to the World Health Organization's (WHO's) recommendations and diet does not adhere to the recommendations for Mexican population. Sex and nutritional status showed a significant association ( $p<0.005)$, as well as blood pressure and nutritional status ( $p<0.05$, Odds Ratio 6.9, Confidence Intervals 3.1-15.4). Conclusions: Children with overweight and obesity had a significant association and a higher risk of developing hypertension than children with low or normal nutritional status that is why the primary prevention should start during the childhood.
\end{abstract}

Key words: Nutritional status. Blood Pressure. Diet. Physical Activity. Children.
Correspondencia:

* José J. Pérez-Miss

E-mail: Joseperez3895@gmail.com
Fecha de recepción: 08-10-2020

Fecha de aceptación: 17-11-2020

DOI: 10.24875/RME.20000097
Disponible en internet: 10-08-2021

Rev Mex Endocrinol Metab Nutr. 2021;8:126-33

2462-4144 / @ 2020 Sociedad Mexicana de Nutricion y Endocrinologia, AC. Publicado por Permanyer. Este es un artículo open access bajo la licencia CC BY-NC-ND (http://creativecommons.org/licenses/by-nc-nd/4.0/). 


\section{INTRODUCCIÓN}

La hipertensión arterial (HTA) en la edad pediátrica es una entidad frecuentemente subdiagnosticada con características propias en cuanto a diagnóstico, etiología y manejo ${ }^{1}$. En México, en la edad escolar, se ha reportado del 3.2 al $16.2 \%$ de presión arterial (PA) elevada, antes conocida como prehipertensión, y del 1 al 5.5\% de HTA, según Rosas Peralta ${ }^{2}$.

Respecto al estado nutricional, la Encuesta Nacional de Salud y Nutrición (ENSANUT) $2016^{3}$ reportó que, en la región sur del país, donde se ubica Yucatán, la prevalencia de sobrepeso y obesidad en la población edad escolar (5-11 años) fue del 17.6 y $13.4 \%$ respectivamente. Actualmente, esta problemática se ha reconocido como uno de los retos más importantes de salud pública debido a su magnitud, reducción en la calidad de vida e incremento del riesgo de padecer enfermedades crónicas no transmisibles ${ }^{4}$.

Estudios mexicanos han encontrado que la dieta de los escolares comprende alimentos no recomendables para consumo cotidiano (productos ultraprocesados y bebidas azucaradas) y también su asociación con sobrepeso y obesidad 5 . Por otro lado, la Organización Mundial de la Salud (OMS) recomienda que se realicen actividades físicas de intensidad moderada a vigorosa al menos una hora al día, con una frecuencia a su vez de tres o más días por semana ${ }^{6}$. Bajo este aspecto, la ENSANUT $2016^{3}$ reportó que solo un $17.2 \%$ de la población entre 10 y 14 años cumple esta recomendación. Asimismo, diversos estudios han encontrado la asociación de la falta de actividad física con el desarrollo de sobrepeso y obesidad ${ }^{7}$.

Estudios longitudinales han relacionado la obesidad infantil con un cambio en la distribución de PA, lo que puede traducirse en HTA en la edad adulta, postulando la importancia de realizar más estudios de investigación sobre esta problemática con la finalidad de disminuir la prevalencia de HTA en edades posteriores ${ }^{8}$. La dieta y actividad física no se han relacionado con el desarrollo de HTA o quizá sigan un camino indirecto por el desarrollo de obesidad, pero dichos mecanismos están aún en investigación ${ }^{9}$.

El propósito del estudio fue conocer la asociación del estado nutricional, dieta, así como la actividad física con las cifras de PA en escolares de 8 a 12 años de tres escuelas primarias de diferentes zonas de la ciudad de Mérida, Yucatán.

\section{MATERIAL Y MÉTODOS}

El presente trabajo formó parte del proyecto: «Intervención integral en niños, niñas escolares y sus familias en el municipio de Mérida» del Consejo Municipal de Salud del Ayuntamiento de Mérida, Yucatán, México. Se realizó un estudio transversal, observacional, analítico y prospectivo.

El universo lo conformaron escolares de todas las primarias públicas del municipio de Mérida y la muestra se eligió de conformidad con los siguientes criterios: a) el ámbito geográfico fue Mérida, Yucatán; b) la zona geográfica se acotó de acuerdo con lo establecido por la Secretaría de Educación del Gobierno del Estado de Yucatán (SEGEY) en zona I sur, zona II centro y zona III norte; c) turno matutino eligiendo una escuela por zona. Se trabajó con todos los escolares de $4 .^{\circ}$ a $6 .^{\circ}$ grado de las tres escuelas primarias seleccionadas durante el primer semestre del año 2018, al ser escuelas públicas de todos los sectores de la ciudad, están representados todos los estratos socioeconómicos, lo que daría representatividad a la muestra.

Asentimiento y consentimiento informado por escrito fueron solicitados cumpliendo los lineamientos éticos establecidos y garantizando la confidencialidad de los datos. Para recabar la información sobre actividad física se utilizó una encuesta exprofeso, mientras que los datos dietéticos se obtuvieron por medio de un cuestionario cualitativo para valorar la frecuencia de consumo de los siete días anteriores a la entrevista. Se incluyeron a todos los escolares entre 8 y 12 años de los grados referidos $\left(4 .^{\circ}\right.$ a $\left.6 .^{\circ}\right)$ y que acudieron a todas las mediciones necesarias durante el estudio: PA, dieta, actividad física y antropometría. 
Las mediciones de peso $(\mathrm{kg})$ y talla $(\mathrm{m})$ se recogieron aplicando la técnica estandarizada de Habitch ${ }^{10}$, se analizaron y clasificaron de acuerdo con el índice de masa corporal (IMC) para edad en bajo peso (z-sco$r e<-2$ ), peso normal ( $z$-score $\geq-2<+1$ ), sobrepeso ( $z$-score $\geq+1<+2$ ) y obesidad ( $z$-score $\geq+2$ ), con el programa WHO Anthro Plus ${ }^{11}$.

Para la medición y evaluación de la PA se siguieron las recomendaciones de la Academia Americana de Pediatría (AAP) para el diagnóstico y manejo de HTA en niños y adolescentes del año $2017^{12}$, y cuya recomendación establece realizar tres mediciones consecutivas, pero separadas por mínimo de una semana entre ellas, para plantear el diagnóstico de PA elevada o hipertensión.

A su vez, la AAP otorga valores de corte nuevos y define: PA normal como una PA sistólica y diastólica menor al percentil 90; PA elevada como algún componente de la PA mayor o igual al percentil 90 pero menor al 95 o, igual o mayor a $120 / 80 \mathrm{mmHg}$ pero menor al percentil 95; HTA grado I como algún componente de la PA mayor o igual al percentil 95 pero menor al percentil 95 más $12 \mathrm{mmHg}$ o, mayor o igual a 130/80 $\mathrm{mmHg}$ pero menor a 139/89 $\mathrm{mmHg}$; HTA grado II cuando algún componente sea mayor o igual al percentil 95 más $12 \mathrm{mmHg}$ o mayor a 140/90 mmHg.

La actividad física se evaluó de acuerdo con las recomendaciones de la $\mathrm{OMS}^{6}$, clasificándola como: nula, menor a una hora al día y mayor o igual a una hora al día, tres o más veces por semana. Respecto a la dieta, para evaluar la cantidad de porciones consumidas se utilizó como referencia las Guías alimentarias y de actividad física en contexto de sobrepeso y obesidad en población mexicana ${ }^{13}$; el consumo de alimentos recomendables y no recomendables para consumo cotidiano ${ }^{14}$ y la ingesta de productos ultraprocesados de acuerdo con el sistema NOVA ${ }^{15}$.

Los datos se procesaron con el paquete estadístico SPSS v. 20. Se realizaron medidas de tendencia central y chi cuadrada de Pearson $\left(c^{2}\right)$ con un $95 \%$ de confianza para determinar la asociación entre las variables estudiadas. Para analizar prevalencia y rango relativo se utilizaron tablas de contingencia, para valorar el riesgo relativo se utilizó la RM.

\section{RESULTADOS}

En total, se trabajó con una plantilla estudiantil de 327 niños de las tres escuelas; se excluyó a 30 estudiantes por no acudir a las tres mediciones necesarias para este estudio, quedando constituido el universo por 297 niños, de los cuales el 48.5\% (144) era del sexo femenino y $51.5 \%$ (153) del sexo masculino. Sin embargo, se observó que en la zona centro y sur de la ciudad predominó el sexo femenino, con el $56.3 \%$ (49) y $58.9 \%$ (33) respectivamente, siendo en la zona norte donde únicamente predominó el sexo masculino, con el 59.8\% (92), contando también con más de la mitad de la plantilla estudiantil total estudiada, el 51.8\% (154/297).

La mediana de edad fue de 10 años (desviación estándar [DE]: \pm 0.96 ); el peso en mujeres tuvo una media de $41.73 \mathrm{~kg}$ (DE: \pm 11.18 ) y en hombres de $41.07 \mathrm{~kg}$ (DE: \pm 12.89 ); en el caso de la talla en mujeres, la media fue de $1.41 \mathrm{~m}$ y en hombres de $1.39 \mathrm{~m}$; el IMC en niñas tuvo una media de $20.55 \mathrm{~kg} / \mathrm{m}^{2}$ (DE: \pm 3.97 ) y en hombres de $20.51 \mathrm{~kg} / \mathrm{m}^{2}$ (DE: \pm 4.53 ).

Las cifras tensionales en relación con el sexo, estado nutricional, edad, actividad física y dieta se exponen en la tabla 1, destacando la presencia de exceso de peso en el $47.2 \%$ (140) de la población, siendo el sexo masculino el de mayor prevalencia, con el $52.1 \%$ (73); también se observa que el 47.5\% (141) realiza al menos una hora de actividad física al día, tres o más veces por semana. Con respecto a la PA, el $2.4 \%$ (7) mantuvo cifras de PA elevada, el $12.1 \%$ (36) permanecieron con hipertensión grado I y el $1 \%$ (3) con hipertensión grado II, siendo el sexo femenino el que mayor prevalencia tuvo, con el $54.3 \%$ (25) del total.

En el análisis por zona escolar, destaca la escuela ubicada en la zona I (sur) de la ciudad de Mérida, la cual presentó la mayor prevalencia de cifras tensionales elevadas, con el $25 \%(14 / 56)$ y $4.7 \%$ (14/297) de total estudiado (Fig. 1). Al contrario, el norte de la ciudad mostró la mayor prevalencia de sobrepeso y obesidad, con el 48.7\% (74/154), en comparación con las otras zonas (Fig. 2), siendo este mismo donde el $51.2 \%$ de sus estudiantes (79/154) siguen las recomendaciones de la OMS sobre actividad física (Fig. 3). 
Tabla 1. Cifras tensionales en relación con el sexo, estado nutricional, edad, actividad física y dieta

\begin{tabular}{|c|c|c|c|c|c|}
\hline & Normal & $\begin{array}{c}\text { Presión arterial } \\
\text { elevada }\end{array}$ & $\begin{array}{c}\text { Hipertensión } \\
\text { grado I }\end{array}$ & $\begin{array}{l}\text { Hipertensión } \\
\text { grado II }\end{array}$ & Total \\
\hline Total & $251(84.5 \%)$ & $7(2.4 \%)$ & $36(12.1 \%)$ & $3(1 \%)$ & $297(100 \%)$ \\
\hline \multicolumn{6}{|l|}{ Sexo } \\
\hline Masculino & $132(44.4 \%)$ & $5(1.7 \%)$ & $15(5.1 \%)$ & $1(.3 \%)$ & $153(51.5 \%)$ \\
\hline Femenino & $119(40.1 \%)$ & $2(0.7 \%)$ & $21(7.1 \%)$ & $2(0.7 \%)$ & $144(48.5 \%)$ \\
\hline \multicolumn{6}{|l|}{ Estado nutricional } \\
\hline Bajo peso & $22(7.4 \%)$ & $0(0 \%)$ & $0(0 \%)$ & $0(0 \%)$ & $22(7.4 \%)$ \\
\hline Normal & $127(42.8 \%)$ & $4(1.3 \%)$ & $4(1.3 \%)$ & $0(0 \%)$ & $135(45.5 \%)$ \\
\hline Sobrepeso & $62(20.9 \%)$ & $1(0.3 \%)$ & $9(3 \%)$ & $1(0.3 \%)$ & $73(24.6 \%)$ \\
\hline Obesidad & $40(13.5 \%)$ & $2(0.7 \%)$ & $23(7.7 \%)$ & $2(0.7 \%)$ & $67(22.6 \%)$ \\
\hline \multicolumn{6}{|l|}{ Edad } \\
\hline 9 años & $52(17.5 \%)$ & $1(0.3 \%)$ & $5(1.7 \%)$ & $1(0.3 \%)$ & 59 (19.9\%) \\
\hline 10 años & $85(28.6 \%)$ & $1(0.3 \%)$ & $8(2.7 \%)$ & $1(0.3 \%)$ & $95(32 \%)$ \\
\hline 11 años & 77 (25.9\%) & $4(1.3 \%)$ & $18(6.1 \%)$ & $1(0.3 \%)$ & $100(33.7 \%)$ \\
\hline 12 años & $37(12.5 \%)$ & $1(0.3 \%)$ & $5(1.7 \%)$ & $0(0 \%)$ & $43(14.5 \%)$ \\
\hline \multicolumn{6}{|l|}{ Actividad física } \\
\hline Nula & $47(15.8 \%)$ & $2(0.7 \%)$ & $10(3.4 \%)$ & $1(0.3 \%)$ & $60(20.2 \%)$ \\
\hline Menor a una hora al día & $81(27.3 \%)$ & $3(1 \%)$ & $11(3.7 \%)$ & $1(0.3 \%)$ & $96(32.3 \%)$ \\
\hline Mayor a una hora al día & $123(41.4 \%)$ & $2(0.7 \%)$ & $15(5.1 \%)$ & $1(0.3 \%)$ & $141(47.5 \%)$ \\
\hline \multicolumn{6}{|l|}{ Dieta } \\
\hline $\begin{array}{l}\text { Consumo de alimentos } \\
\text { ultraprocesados }\end{array}$ & $251(84.5 \%)$ & $7(2.4 \%)$ & $36(12.1 \%)$ & $3(1 \%)$ & $297(100 \%)$ \\
\hline $\begin{array}{l}\text { Consumo de alimentos } \\
\text { y bebidas saludables }\end{array}$ & $251(84.5 \%)$ & 7 (2.4\%) & $36(12.1 \%)$ & $3(1 \%)$ & $297(100 \%)$ \\
\hline
\end{tabular}

Finalmente, al evaluar la dieta se observó que las porciones de los alimentos recomendables para consumo cotidiano se encuentran por debajo de lo establecido por la Academia Nacional de Medicina de México $(\mathrm{ANM})^{13}$, donde el consumo promedio de vasos de agua al día fue de 5 , el de leche sin sabor 1 porción, y el de frutas-verduras de 2 porciones, siendo la recomendación diaria de 8, 4 y 3 porciones al día respectivamente. Ahora bien, se observó que el promedio general de consumo de alimentos ultraprocesados al día fue de 2 porciones, cuando lo recomendado es lo mínimo posible o cero.

Se encontró asociación estadísticamente significativa entre el sexo masculino y la presencia de sobrepeso/obesidad (RM: 1.04, IC: 0.665-1.654; $\mathrm{p}<0.005$ ).
Asimismo, se encontró una asociación significativa entre PA elevada e hipertensión con el sobrepeso $u$ obesidad (RM: 6.9, IC: 3.1-15.4; $\mathrm{p}<0.05$ ), sin embargo, no se relacionaron significativamente con las otras variables estudiadas respectivamente. Los resultados del análisis de asociación y significancia estadística se presentan en la tabla 2.

\section{DISCUSIÓN}

Si bien la población del presente estudio es pequeña, llama la atención el mayor porcentaje de hipertensión encontrado (13.1\%), comparado con 


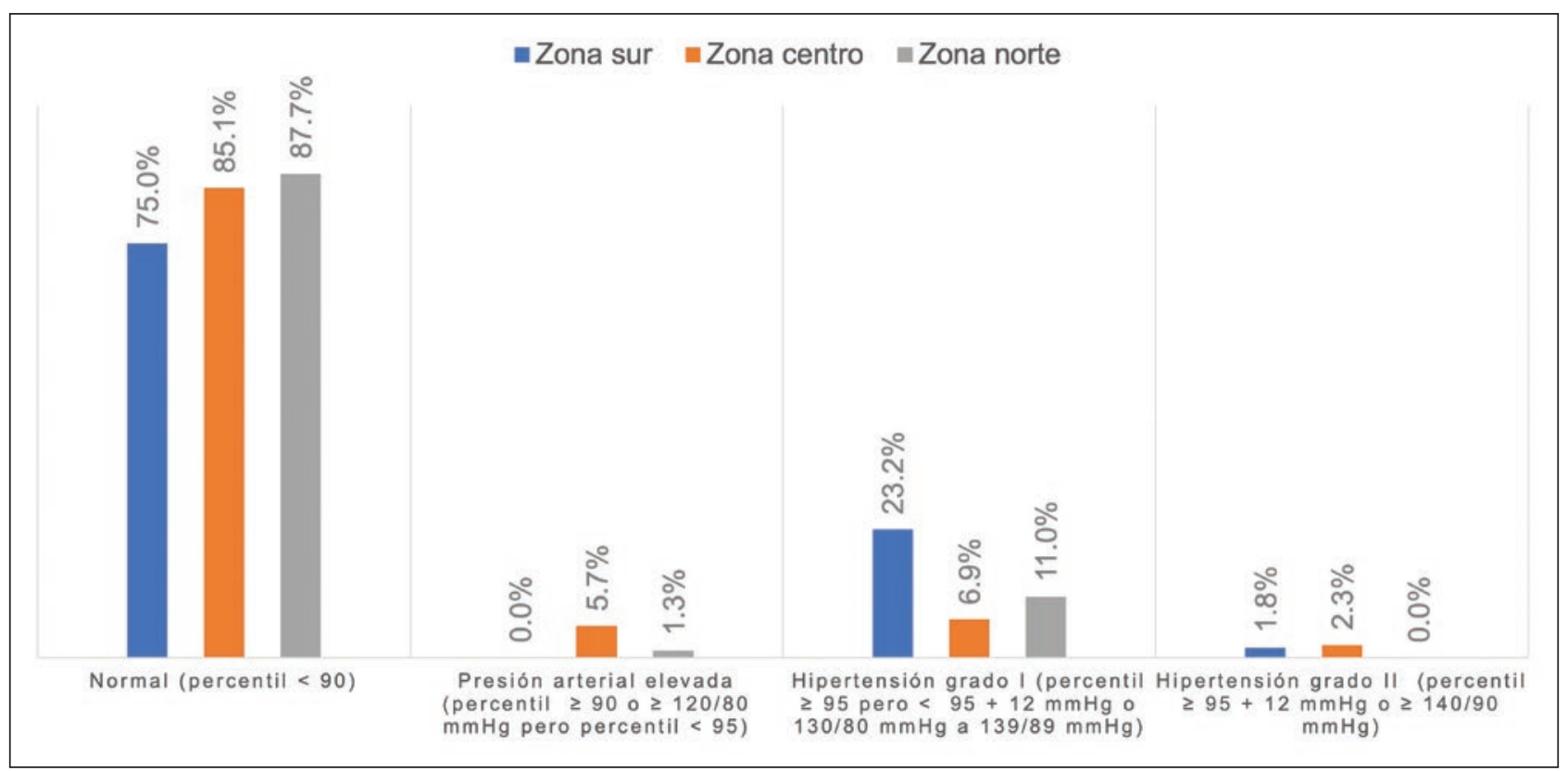

Figura 1. Prevalencia de cifras tensionales en escolares de 8 a 12 años de tres escuelas estratificadas por zonas de la ciudad de Mérida, Yucatán. Las barras indican la prevalencia encontrada para cada clasificación de presión arterial en la respectiva escuela que se tomó como representativa para cada una de las tres zonas (según la Secretaría de Educación del Gobierno del estado de Yucatán) en la que se encuentra dividida la ciudad de Mérida, Yucatán. Nótese la prevalencia de niños con cifras coincidentes a hipertensión arterial grado I, comparado con las prevalencias de presión arterial elevada.

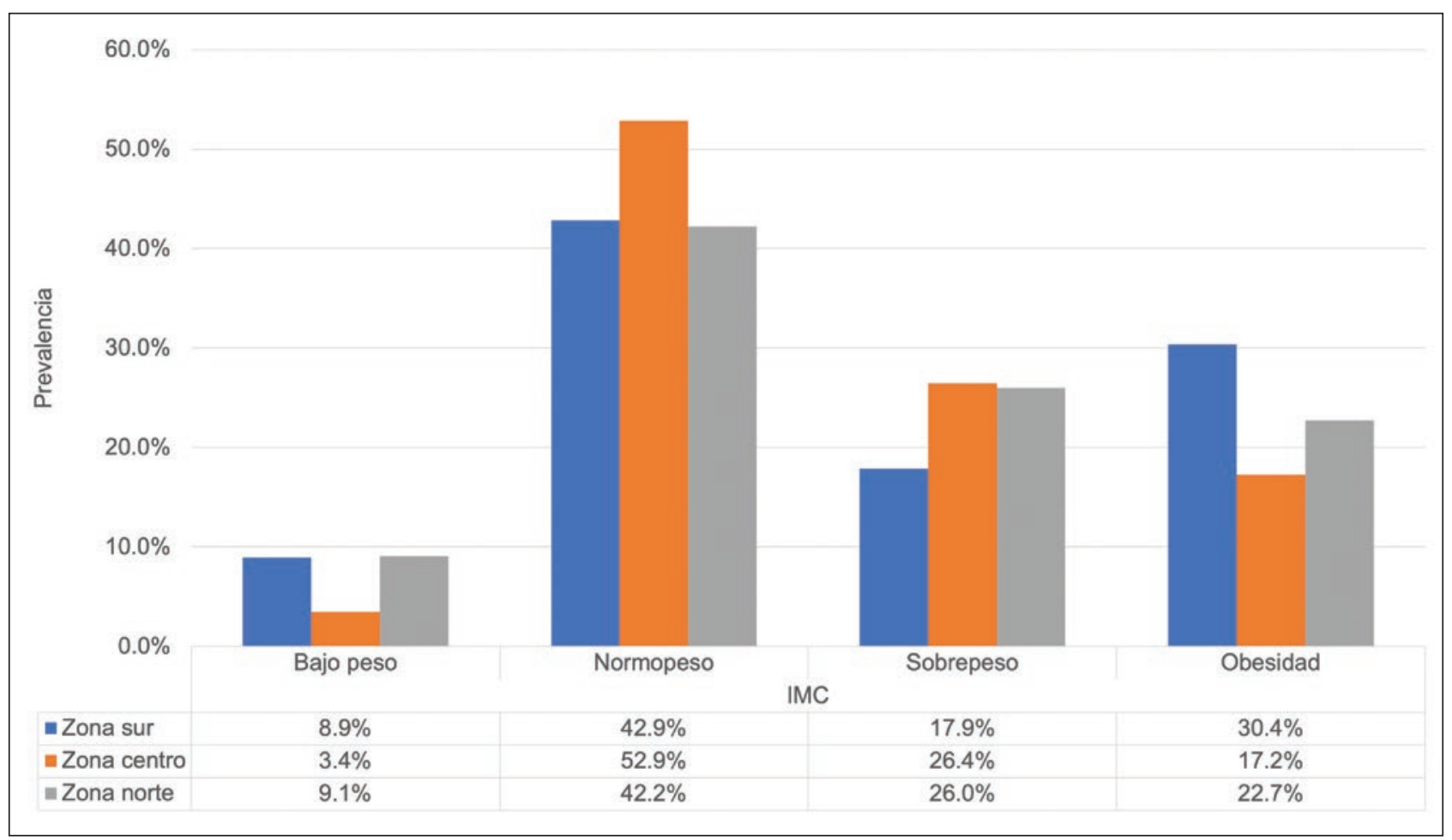

Figura 2. Prevalencia de estado nutricional en escolares de 8 a 12 años de tres zonas escolares de la ciudad de Mérida, Yucatán. Las barras indican la prevalencia encontrada para cada clasificación del estado nutricional con base en el índice de masa corporal (IMC) en la respectiva escuela que se tomó como representativa para cada una de las tres zonas en la que se encuentra dividida la ciudad de Mérida, Yucatán. Nótese la alta prevalencia conjunta de sobrepeso y obesidad que conforman cerca de la mitad de los niños estudiados. 


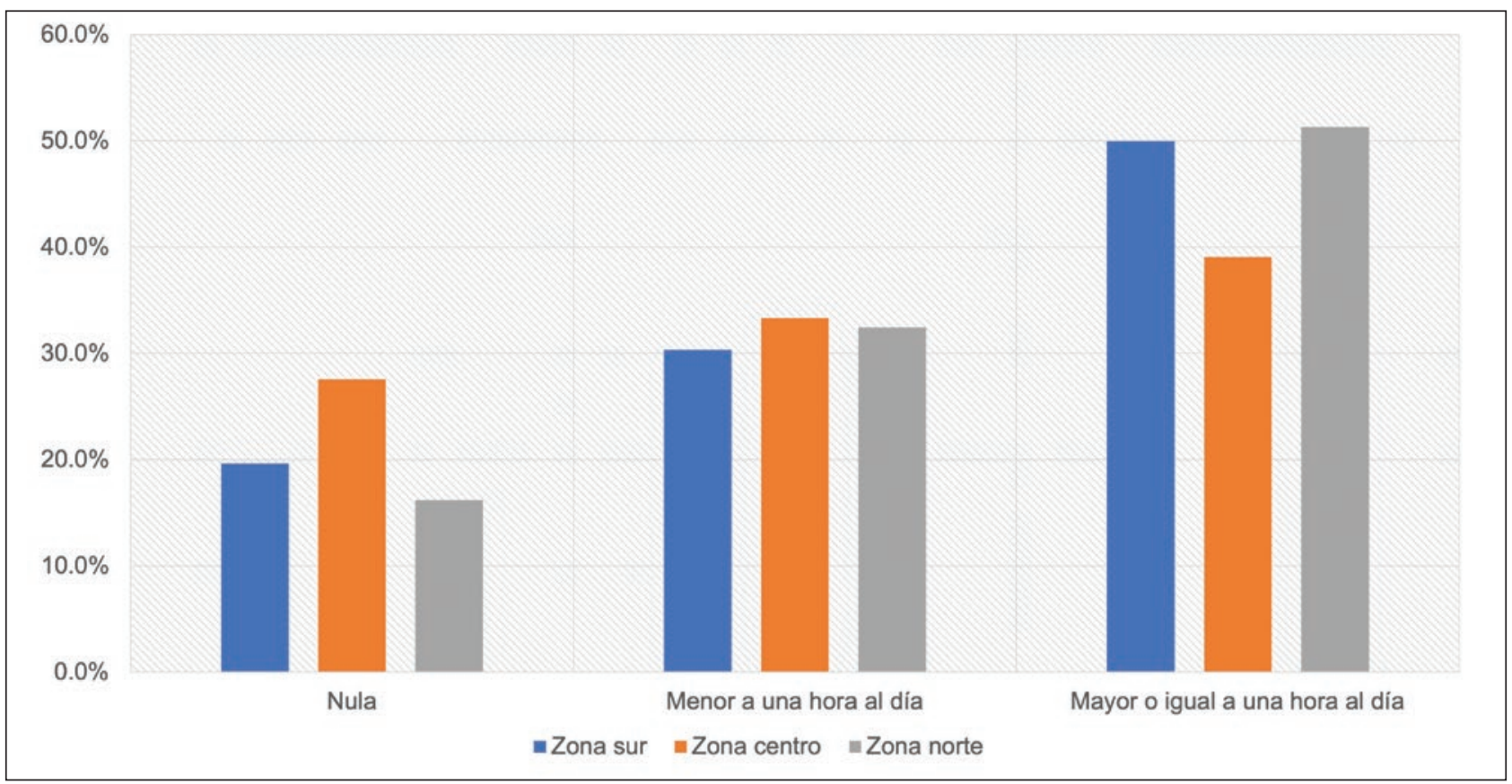

Figura 3. Prevalencia de la actividad física realizada por escolares de 8 a 12 años de tres zonas escolares de la ciudad de Mérida, Yucatán. Las barras indican la prevalencia encontrada para la clasificación del tiempo de actividad física realizada con base en las recomendaciones de la Organización Mundial de la Salud. También se muestra la prevalencia encontrada en cada una de las tres escuelas primarias públicas, las cuales representaron cada zona de la ciudad de Mérida, Yucatán.

Tabla 2. Asociación de los factores estudiados (estado nutricional, dieta, actividad física) con las cifras de presión arterial en niños escolares, de la ciudad de Mérida, Yucatán. Se puede apreciar el riesgo casi siete veces mayor de los niños con sobrepeso u obesidad de desarrollar cifras tensionales elevadas para su edad. Igualmente se aprecia la asociación significativa entre el sexo, en este caso masculino, con el sobrepeso u obesidad. También la asociación significativa entre el sobrepeso y obesidad y la presión arterial elevada o hipertensión

\begin{tabular}{lcccc}
\hline Asociaciones & Razón de momios & Chi cuadrada & $p$ & $\begin{array}{c}\text { Intervalos de } \\
\text { confianza al 95\% }\end{array}$ \\
\hline Sexo y Sob/Ob & 1.04 & 12.855 & $0.005^{*}$ & $0.665-1.654$ \\
\hline Sexo y PAE/H & 0.757 & 3.022 & 0.388 & $0.403-1.423$ \\
\hline Edad y Sob/Ob & -- & 8.659 & 0.469 & -- \\
\hline Edad y PAE/H & -- & 8.279 & 0.506 & $3.109-15.488$ \\
\hline Sob/Ob y PAE/H & 6.939 & 52.646 & $0.001^{*}$ & $0.787-2.840$ \\
\hline AM y PAE/H & 1.495 & 3.101 & 0.125 & $1.103-1.519$ \\
\hline Dieta y PAE/H & 1.294 & 4.152 & 0.279 & $0.616-1.533$ \\
\hline AM y Sob/Ob & 0.971 & 1.976 & 0.148 & $1.538-2.854$
\end{tabular}

*Estadísticamente significativo.

Sob/Ob: sobrepeso y obesidad; PAE/H: presión arterial elevada e hipertensión; AM: actividad física.

un $5.5 \%$ reportado por Rosas ${ }^{2}$. Igualmente, en Yucatán se han realizado pocos estudios para evaluar la prevalencia de HTA en la edad pediátrica siendo Saury ${ }^{16}$, quien encontró que la prevalencia de hipertensión fue del $6.1 \%$ en una población rural, cifras por debajo de nuestros hallazgos, lo que podría sugerir que el contexto (urbano vs. rural) pudiera influir en los estilos de vida. 
En 2018, Peraza ${ }^{17}$, en una escuela primaria de Mérida, encontró un $15.7 \%$ de prevalencia para PA elevada e hipertensión, similar a lo encontrado en este estudio (15.5\%), el cual abarcó tres zonas diferentes de la ciudad, sin embargo, al evaluar individualmente cada zona, se encontró una mayor prevalencia en el sur (25\%) de la ciudad. Tanto el presente estudio como los dos últimos referidos muestran asociación entre PA y estado nutricional. En cuanto al riesgo relativo de presentar hipertensión, nuestros datos nuevamente se acercan a lo encontrado por Pera$\mathrm{za}^{17}$ (6.9 vs. 5.6 respectivamente) y llama la atención que el riesgo en la zona rural sea 10 veces mayor ${ }^{16}$.

En relación con los factores asociados a hipertensión, se tiene el sobrepeso y obesidad. Al respecto, en este estudio la prevalencia conjunta fue del $47.2 \%$, cifra superior a la reportada por la ENSANUT $2018^{18}$, cuya prevalencia conjunta nacional fue del $35.6 \%$, mostrando una diferencia notoria en nuestra región. Otro factor asociado es la realización de actividad física, donde el $47.5 \%$ de los estudiantes siguen las recomendaciones de la OMS, contrastando con el 17.5\% reportado por la ENSANUT-MC 2016³. Una posible explicación puede ser la asistencia a actividades deportivas extraescolares en algunas zonas urbanas.

En cuanto a la ingesta de alimentos no recomendables para consumo cotidiano, esta fue baja, coincidiendo nuevamente con lo reportado para la región sur del país en la ENSANUT 2018 ${ }^{18}$. También se concluye que la ingesta de alimentos recomendables es insuficiente para cumplir con las recomendaciones de la $\mathrm{ANM}^{13}$. Los resultados fueron presentados a las autoridades educativas, así como padres y madres de familia.

Dentro de las limitantes encontradas, hay que señalar que se consideró un tamaño de muestra piloto estadístico, lo que limitó la población estudiada, sin embargo, los resultados significativos encontrados sugieren la realización de investigaciones con una mayor población para confirmar nuestros hallazgos y con esto desarrollar un plan de intervención para la problemática estudiada. Otra limitante fue obtener datos dietéticos únicamente cualitativos, y no cuantitativos (como el recordatorio de 24 horas), lo que impidió conocer la cantidad de energía consumida por los escolares.

\section{CONCLUSIONES}

Las cifras tensionales elevadas se asocian significativamente con sobrepeso y obesidad (RM: 6.9, IC: 3.1-15.4; $\mathrm{p}<0.05)$. De la misma manera, los escolares con sobrepeso y obesidad tienen un riesgo 6.9 veces mayor de presentar HTA que los niños eutróficos. Existe una alta prevalencia de sobrepeso y obesidad en la población escolar de Mérida, Yucatán comparada con el promedio nacional. La aparente baja ingesta de alimentos ultraprocesados o actividad física adecuada no mostraron asociación con PA elevada o hipertensión, por lo que la prevención primaria de hipertensión desde la infancia mediante la toma periódica de PA es imperativa para reducir la incidencia a largo plazo de complicaciones cardiovasculares en la vida adulta.

\section{AGRADECIMIENTOS}

Al Ayuntamiento de Mérida 2015-2018, al Consejo Municipal de Salud, al equipo de la Subdirección Municipal de Salud, así como a la Secretaría de Educación del Gobierno del Estado de Yucatán, por su apoyo en la realización del presente trabajo, pero sobre todo a los niños y niñas que colaboraron desinteresadamente en este estudio.

\section{FINANCIAMIENTO}

La presente investigación no recibió ninguna beca especifica de agencias de los sectores públicos, comercial o sin ánimo de lucro.

\section{CONFLICTO DE INTERESES}

Los autores declaran no tener ningún conflicto de intereses. 
RESPONSABILIDADES ÉTICAS

Protección de personas y animales. Los autores declaran que para esta investigación no se han realizado experimentos en seres humanos ni en animales.

Confidencialidad de los datos. Los autores declaran que han seguido los protocolos de su centro de trabajo sobre la publicación de datos de pacientes.

Derecho a la privacidad y consentimiento informado. Los autores han obtenido el consentimiento informado de los pacientes y/o sujetos referidos en el artículo.

\section{BIBLIOGRAFÍA}

1. De la Cerda-Ojeda F, Herrero-Hernando C. Hipertensión arterial en niños y adolescentes. Protoc Diagn Ter Pediatr. 2014;1:171-89.

2. Rosas-Peralta M, Medina-Concebida LE, Borrayo-Sánchez G, MadridMiller A, Ramírez-Arias E, Pérez-Rodríguez G. Hipertensión arterial sistémica en el niño y adolescente. Rev Med Inst Mex Seguro Soc. 2016;54(S1):52-66.

3. Instituto Nacional de Salud Pública. Encuesta Nacional de Salud y Nutrición 2016. Informe final de resultados [Internet]. México: Instituto Nacional de Salud Pública, Secretaría de Salud; 2016 [consultada: 15/03/2019]. Disponible en: http://transparencia.insp.mx/2017/auditorias-insp/12701_Resultados_Encuesta_ENSANUT MC2016.pdf

4. García-García E, de la Llata-Romero D, Kaufer-Horwitz M, Tusié-Luna MT, Calzada-León R, Vázquez-Velázquez V, et al. La obesidad y el síndrome metabólico como problema de salud pública. Una reflexión. Salud Publ Mex. 2008;50:530-47.

5. Cárdenas-Villalvazo A, Lupita-Barreto A, Martínez-Moreno G, AguileraCervantes VG, Acosta-Aguilar P, Ortiz-Gómez L, et al. Hábitos de alimen- tación en el hogar y la escuela como factores de obesidad infantil en Cd. Guzmán, Jalisco. Rev Mex Invest Psic. 2017;4(S1):59-70.

6. Organización Mundial de la Salud. Recomendaciones mundiales sobre la actividad física para la salud [Internet]. Ginebra; 2010 [consultada: 15/06/2018]. Disponible en: http://whqlibdoc.who.int/publications/2010/9789243599977_spa.pdf

7. Ramírez-Izcoa A, Sánchez-Sierra LE, Mejía-Irías C, Izaguirre González Al Alvarado-Avilez C, Flores-Moreno R, et al. Prevalencia y factores asociados a sobrepeso y obesidad infantil en escuelas públicas y privadas de Tegucigalpa, Honduras. Rev Chil Nutr. 2017;44(2):161-9.

8. Bao W, Threefoot SA, Srinivasan SR, Berenson GS. Essential hypertension predicted by tracking of elevated blood pressure from childhood to adulthood: the Bogalusa Heart Study. Am J Hypertens. 1995;8:657-65.

9. Dorresteijn J, Visseren F, Spiering W. Mechanisms linking obesity to hypertension. Obes Rev. 2012;13(1):17-26.

10. Habicht JP. Estandarización de métodos epidemiológicos cuantitativos sobre el terreno. Boletín de la Oficina Sanitaria Panamericana. 1974;76: 375-84.

11. Organización Mundial de la Salud. Patrones de crecimiento infantil [lnternet]. Ginebra; 2006 [consultada 15/06/2018]. Disponible en: https:// www.who.int/childgrowth/standards/es/

12. Flynn JT, Kaelber DC, Baker-Smith CM, Blowey D, Carroll AE, Daniels SR, et al. Clinical practice guideline for screening and management of high blood pressure in children and adolescents. Pediatrics. 2017;140(3):e20171904.

13. Bonvecchio A, Fernández AC, Plazas M, Kaufer M, Pérez AB, Rivera JA. Guías alimentarias y de actividad física en contexto de sobrepeso y obesidad en la población mexicana [Internet]. México: Academia Nacional de Medicina; 2015 [consultada: 15/06/2018]. Disponible en: https:// www.insp.mx/epppo/blog/3878-guias-alimentarias.html

14. Gaona-Pineda EB, Martínez-Tapia B, Arango-Angarita A, ValenzuelaBravo D, Gómez-Acosta LM, Shamah-Levy T, et al. Consumo de grupos de alimentos y factores sociodemográficos en población mexicana. Salud Publ. Mex. 2018;60(3):272-82.

15. Organización Panamericana de la Salud, Organización Mundial de la Salud. Alimentos y bebidas ultraprocesados en América Latina: tendencias, efecto sobre la obesidad e implicaciones para las políticas públicas [Internet]. Washington DC: Organización Panamericana de la Salud, Organización Mundial de la Salud; 2015 [consultada: 15/06/2018]. Disponible en: http://iris.paho.org/xmlui/handle/123456789/7698

16. Saury-Paredes LA. Prevalencia de cifras tensionales elevadas y su asociación con el estado nutricional en niños de entre 5 y 11 años de Nahbalam, Yucatán. Gac Med Mex. 2016;152(5):640-44.

17. Peraza-López EE, Zapata-Peraza AL. Asociación de la obesidad abdominal y alteraciones de la presión arterial con estrategias de afrontamiento ante la carestía alimentaria en niños en edad escolar. Rev Biomed. 2018;29(3):51-9.

18. Instituto Nacional de Salud Pública. Encuesta Nacional de Salud y Nutrición 2018. Presentación de resultados [Internet] Cuernavaca, México: Instituto Nacional de Salud Pública; 2018 [consultado: 13/03/2019]. Disponible en: https://ensanut.insp.mx/encuestas/ensanut2018/doctos/ informes/ensanut_2018_presentacion_resultados.pdf 